\title{
Nota Científica \\ Propriedades da madeira de Eucalyptus benthamii para produção de energia
}

\author{
Dimas Agostinho Silva ${ }^{1 *}$, Bruna Verediana Müller², Elaine Cristina Kuiaski', Elder Eloy ${ }^{3}$, Alexandre Behling ${ }^{1}$, Cecilia Moura Colaço $^{1}$ \\ ${ }^{1}$ Universidade Federal do Paraná, CIFLOMA, Av. Pref. Lothário Meissner, 632, CEP 80210-170, Curitiba, PR, Brasil \\ ${ }^{2}$ Instituto Federal de Educação, Ciência e Tecnologia Catarinense, Estrada do Redentor, 5665, CEP 89163-356, Rio do Sul, SC, Brasil \\ ${ }^{3}$ Universidade Federal de Santa Maria, BR 386, Km 40, s/n, Linha Sete de Setembro, CEP 98400-000, Frederico Westphalen, RS, Brasil
}

*Autor correspondente:

dimas.agostinho.silva@gmail.com

Termos para indexação:

Eucalipto

Dendroenergia

Biomassa florestal

Index terms:

Eucalyptus

Wood energy

Forest biomass

Histórico do artigo:

Recebido em 25/03/2014

Aprovado em 09/12/2015

Publicado em 31/12/2015

doi: 10.4336/2015.pfb.35.84.677

\begin{abstract}
Resumo- Este estudo objetivou avaliar o potencial energético da madeira de Eucalyptus benthamii Maiden et Cambage. As amostras foram coletadas no município de Cerro Negro, SC, onde foram selecionadas 5 árvores, sendo retiradas amostras do fuste a $0 \%$, $25 \%, 50 \%, 75 \%$ e $100 \%$ da altura comercial. As análises realizadas foram densidade básica, poder calorífico superior, composição química elementar, análise química imediata (teores de materiais voláteis, carbono fixo e cinzas), poder calorífico inferior, densidade energética, estoque de carbono e produção energética. As variáveis físicas e químicas estudadas e o potencial energético da madeira não apresentaram diferenças ao longo do fuste.
\end{abstract}

\section{Properties of Eucalyptus benthamii wood for energy production}

\begin{abstract}
The objective of this study was to evaluate the energy potential of Eucalyptus benthamii Maiden et Cambage wood. The samples were collected in the municipality of Cerro Negro, Santa Catarina State, Brazil. Samples were collected from 5 trees at $0 \%, 25 \%, 50 \%, 75 \%$ and $100 \%$ of commercial height. It was determined basic density, high calorific value, elemental composition, immediate chemical analysis, lower calorific value, energy density, carbon storage and energy production. The physical and chemical variables studied and energy potential of wood did not present differences along the stem.
\end{abstract}

A busca por fontes alternativas de energia limpa e renovável vem resultando em um aumento na demanda da biomassa florestal, que é uma opção à queima de combustíveis fósseis (fonte energética não-renovável), uma vez que as árvores têm o potencial de capturar e armazenar em sua biomassa o carbono existente na atmosfera. A energia de biomassa também é considerada como uma alternativa econômica eficaz e viável para suprir as demandas em diversos setores da sociedade, apresentando cada vez mais importância na matriz energética nacional.
A espécie Eucalyptus benthamii Maiden et Cambage é originária da Austrália, com maior ocorrência nas regiões do sudoeste de Sydney, nas planícies de Nepean River e seus afluentes. Foi introduzida no Brasil pela Embrapa Florestas (Nisgoski et al., 1998), se destacando por apresentar uma alta produtividade e adaptabilidade ao clima da região Sul. Em Santa Catarina, Higa \& Pereira (2003) registraram em plantios experimentais de E. benthamii com 2 e 3 anos bom crescimento e resistência a geadas. 
Deste modo, este estudo teve como objetivo determinar o potencial energético da madeira de $E$. benthamii com o intuito de avaliar sua viabilidade para produção de energia.

A coleta de amostras foi realizada em maio de 2011, em um plantio comercial de E. benthamii, em espaçamento 2,5 m x 2,0 m com cinco anos, localizado no distrito de São Jorge, município de Cerro Negro, SC. O município está a $996 \mathrm{~m}$ de altitude, sendo o clima classificado como mesotérmico úmido, com verão ameno e temperatura média entre $5^{\circ} \mathrm{C}$ e $18{ }^{\circ} \mathrm{C}$ (Cerro Negro, 2015).

Foram selecionadas, aleatoriamente, cinco árvores de $E$. benthamii, sendo retirados discos de madeira em diferentes posições ao longo do fuste $(0 \%, 25 \%, 50 \%$, $75 \%$ e $100 \%$ da altura comercial), totalizando 5 discos por árvore. Posteriormente, este material foi moído em moinho de facas, com peneira de 200 mesh, visando à obtenção de um material mais fino e uniforme.

Avaliou-se densidade básica, poder calorífico superior, poder calorífico inferior, densidade energética, composição química imediata (teor de materiais voláteis, teor de cinzas, teor de carbono fixo), composição química elementar, estoque de carbono e produção energética por fuste.

A análise da densidade básica (DB), realizada nas 5 posições mensuradas ao longo do fuste de cada árvore, foi determinada de acordo com a norma técnica NBR 11941 (Associação Brasileira de Normas Técnicas, 2003).

Para a determinação do poder calorífico superior (PCS), em Kcal Kg ${ }^{-1}$, foi utilizada bomba calorimétrica digital modelo C5000 Cooling System, IKA Werke, com princípio de funcionamento dinâmico, de acordo com a norma técnica NBR 8633 (Associação Brasileira de Normas Técnicas, 1984).

Os teores de materiais voláteis (TV), teor de cinzas (TC), teor de carbono fixo (CF) foram determinados de acordo com a norma NBR 8112 (Associação Brasileira de Normas Técnicas, 1983).

Para a realização da análise de composição química elementar (CHNS-O) foram utilizados $2,5 \mathrm{mg}( \pm 0,5)$ do material com granulometria menor que 200 mesh, no equipamento CHNS-O Flash EA 1112 Series.

A massa seca total (MST), que corresponde à biomassa da madeira do fuste das árvores, foi estimada em função do volume e da densidade básica, por meio da expressão 1 .

$$
\mathrm{MST}=\mathrm{VT} * \mathrm{DB}
$$

Em que: $\mathrm{MST}=$ massa seca total do fuste $(\mathrm{kg}) ; \mathrm{VT}=$ o volume total sem casca do fuste $\left(\mathrm{m}^{3}\right) ; \mathrm{DB}=$ densidade básica média $\left(\mathrm{kg} \mathrm{m}^{-3}\right)$.

O poder calorífico inferior (PCI) foi calculado a partir da expressão criada por Doat (1977), considerando os valores de poder calorífico superior e teor de hidrogênio, conforme a expressão 2.

$$
\mathrm{PCI}=\mathrm{PCS}-600 * 9 * \mathrm{H} / 100
$$

Em que: $\mathrm{PCI}=$ poder calorífico inferior $\left(\mathrm{kcal} \mathrm{kg}^{-1}\right)$; $\mathrm{PCS}=$ poder calorífico superior $\left(\mathrm{kcal} \mathrm{kg}^{-1}\right) ; \mathrm{H}=$ teor de hidrogênio (\%).

A densidade energética (DE) foi calculada baseada nos valores da densidade básica e poder calorífico superior, conforme a expressão 3.

$$
\mathrm{DE}=\mathrm{DB} * \mathrm{PCS}
$$

Em que: $\mathrm{DE}=$ densidade energética $\left(\mathrm{kcal} \mathrm{m}^{-3}\right) ; \mathrm{DB}$ $=$ densidade básica $\left(\mathrm{kg} \mathrm{m}^{-3}\right) ; \mathrm{PCS}=$ poder calorífico superior $\left(\mathrm{kcal} \mathrm{kg}^{-1}\right)$.

$\mathrm{O}$ estoque de carbono (EC) presente nos fustes foi estimado conforme a expressão 4.

$$
\mathrm{EC}=\mathrm{MS} * \mathrm{TCE}
$$

Em que: $\mathrm{EC}=$ estoque de carbono no fuste $\left(\mathrm{kg}_{\mathrm{fuste}} \mathrm{e}^{-1}\right)$; $\mathrm{MS}=$ massa seca total do fuste $(\mathrm{kg}) ; \mathrm{TCE}=$ teor de carbono elementar (\%).

A produção energética $(\mathrm{PE})$, que corresponde à quantidade disponibilizada de calor para cada fuste, foi obtida em função da massa seca total do fuste e do respectivo poder calorífico superior, conforme a expressão 5 .

$$
\mathrm{PE}=\mathrm{MS} * \mathrm{PCS}
$$

Em que: $\mathrm{PE}=$ produção energética $\left(\mathrm{kcal}\right.$ fuste $\left.{ }^{-1}\right) ; \mathrm{MS}$ $=$ massa seca total do fuste $(\mathrm{kg}) ; \mathrm{PCS}=$ poder calorífico superior $\left(\mathrm{kcal} \mathrm{kg}^{-1}\right)$.

Foram determinados os valores médios, desvio padrão e coeficiente de variação para todas as características estudadas. Para as análises estatísticas utilizou-se o delineamento inteiramente casualizado, com cinco tratamentos (diferentes alturas) e cinco repetições (árvores). Foi realizada a análise de variância, em nível de $95 \%$ de probabilidade, e a análise de correlação linear simples de Pearson.

Os valores médios das propriedades da madeira de $E$. benthamii, bem como seus respectivos valores de desvio padrão e coeficiente de variação estão apresentados na Tabela 1. 
Tabela 1. Valores médios, desvio padrão e coeficiente de variação das características da madeira de Eucalyptus benthamii.

\begin{tabular}{lccc}
\hline \multicolumn{1}{c}{ Característica } & Média & $\begin{array}{c}\text { Desvio } \\
\text { padrão }\end{array}$ & $\begin{array}{c}\text { Coeficiente } \\
\text { de variação }\end{array}$ \\
\hline Densidade básica $\left(\mathrm{g} \mathrm{cm}^{-3}\right)$ & 0,471 & 0,032 & 6,86 \\
Densidade energética $\left(\mathrm{Mcal} \mathrm{m}^{-3}\right)$ & 2206,9 & 164,1 & 7,43 \\
Poder calorífico superior (kcal & 4679,7 & 116,3 & 2,4 \\
$\left.\mathrm{~kg}^{-1}\right)$ & & 116,5 & 2,6 \\
Poder calorífico inferior (kcal kg $\left.{ }^{-1}\right)$ & 4379,1 & 1,3 & 8,82 \\
Teor de carbono fixo (\%) & 15,78 & 1,3 & 1,64 \\
Teor de materiais voláteis (\%) & 83,98 & 0,15 & 63,96 \\
Teor de cinzas (\%) & 0,244 & 0,23 & 0,14 \\
Nitrogênio (\%) & 1,63 & 1,40 & 0,03 \\
Carbono (\%) & 46,68 & 0,20 & 0,03 \\
Hidrogênio (\%) & 5,94 & 0,10 & 0,89 \\
Enxofre (\%) & 0,11 & 1,49 & 0,03 \\
Oxigênio (\%) & 45,64 & 4,91 & 0,17 \\
Relação carbono/nitrogênio (C/N) & 29,23 & 0,14 & 0,02 \\
Relação carbono/hidrogênio (C/H) & 7,87 & 14,54 & 25,87 \\
Estoque de carbono (kg fuste $\left.{ }^{-1}\right)$ & 56,22 & 119964,8 & 21,39 \\
Produção energética (kcal fuste-1) & 560825,8 & \\
\hline & & & \\
\hline
\end{tabular}

O resumo da análise de variância realizada para a densidade básica, densidade energética, teor de carbono fixo, teor de materiais voláteis, teor de cinzas, poder calorífico superior e inferior da madeira ao longo do fuste está apresentado na tabela 2.

Os valores encontrados para as características energéticas da madeira não apresentaram variação significativa ao longo do fuste das árvores. O potencial energético da madeira também não apresentou diferença em função da altura do fuste da árvore. Isso indica que a amostragem de madeira no fuste pode ser realizada em qualquer altura, não sendo necessária a amostragem em diferentes posições.

Pela análise de correlação linear simples de Pearson (Tabela 3) observou-se correlações positivas mais elevadas entre a densidade básica e a densidade energética e entre o poder calorífico superior com o poder calorífico inferior. Correlações negativas foram verificadas para o teor de materiais voláteis com o teor de carbono fixo e com o teor de cinzas.

Tabela 2. Resumo da análise de variância realizada para as características determinadas em diferentes posições ao longo do fuste.

\begin{tabular}{lcccccccc}
\hline \multirow{2}{*}{ FV } & \multirow{2}{*}{ GL } & DB & DE & CF & MV & TC & PCS & PCI \\
\hline Posição & 4 & $0,0022^{\text {ns }}$ & $63375,434^{\text {ns }}$ & $3,915^{\text {ns }}$ & $3,848^{\text {ns }}$ & $0,0205^{\text {ns }}$ & $17050,66^{\text {ns }}$ & $17050,66^{\text {ns }}$ \\
$\mathrm{R}^{2}$ & & 0,35 & 0,35 & 0,34 & 0,34 & 0,34 & 0,21 & 0,20 \\
CV $(\%)$ & & 6,05 & 7,00 & 7,87 & 1,46 & 6,92 & 2,42 & 2,67 \\
\hline
\end{tabular}

Em que: $\mathrm{FV}=$ fator de variação; $\mathrm{GL}=$ graus de liberdade; $\mathrm{R}^{2}=$ coeficiente de determinação; $\mathrm{CV}=$ coeficiente de variação (\%); $\mathrm{DB}=$ densidade básica; $\mathrm{DE}=$ densidade energética; $\mathrm{CF}=$ teor de carbono fixo; $\mathrm{MV}=$ teor de materiais voláteis; $\mathrm{TC}=$ teor de cinzas; $\mathrm{PCS}$ = poder calorífico superior; $\mathrm{PCI}=$ poder calorífico inferior; $\mathrm{ns}=$ não significativo a $5 \%$ de probabilidade pelo teste de $\mathrm{F}$.

Tabela 3. Análise da correlação linear simples de Pearson entre as características determinadas em amostras de Eucalyptus benthamii.

\begin{tabular}{|c|c|c|c|c|c|c|c|}
\hline & DB & DE & CF & MV & TC & PCS & PCI \\
\hline DB & 1 & & & & & & \\
\hline $\mathrm{DE}$ & $0,9644^{*}$ & 1 & & & & & \\
\hline $\mathrm{CF}$ & $0,0083^{\mathrm{ns}}$ & $0,1226^{\mathrm{ns}}$ & 1 & & & & \\
\hline MV & $0,0056^{\mathrm{ns}}$ & $-0,1046^{\mathrm{ns}}$ & $-0,9930^{*}$ & 1 & & & \\
\hline $\mathrm{TC}$ & $-0,027^{\mathrm{ns}}$ & $0,0716^{\mathrm{ns}}$ & $0,6490 *$ & $-0,6636^{*}$ & 1 & & \\
\hline PCS & $0,3314^{\mathrm{ns}}$ & $0,5689 *$ & $0,4044^{*}$ & $-0,3857^{\mathrm{ns}}$ & $0,3370^{\mathrm{ns}}$ & 1 & \\
\hline PCI & $0,3274^{\mathrm{ns}}$ & $0,5650 *$ & $0,4141 *$ & $-0,3961^{\mathrm{ns}}$ & $0,3515^{\mathrm{ns}}$ & $0,9982 *$ & 1 \\
\hline
\end{tabular}

Em que: $\mathrm{DB}=$ densidade básica; $\mathrm{DE}=$ densidade energética; $\mathrm{CF}=$ teor de carbono fixo, $\mathrm{MV}=$ teor de materiais voláteis; $\mathrm{TC}=$ teor de cinzas; $\mathrm{PCS}=$ poder calorífico superior; $\mathrm{PCI}=$ poder calorífico inferior; $*$ e ns $=$ significativo e ns não significativo a $95 \%$ de probabilidade conforme o teste $t$, respectivamente. 
Dentre as características dendroenergéticas, como as que possuem maior relevância, destacam-se a densidade básica, o poder calorífico (superior e inferior), a composição química (elementar e imediata) e a umidade. A densidade da madeira é alterada pelas diferentes propriedades anatômicas e químicas da madeira, sendo positivamente relacionada ao conteúdo calórico da madeira por unidade de volume (Quirino et al., 2004). A densidade básica média da madeira de $E$. benthamii determinada foi de $0,471 \mathrm{~g} \mathrm{~cm}^{-3}$ (Tabela 1), não apresentando diferença em relação ao relatado por Lima et al. (2011), de $0,475 \mathrm{~g} \mathrm{~cm}^{-3}$ em indivíduos de seis anos no município de Guarapuava, PR. No entanto, Pereira et al. (2000) observaram um valor de $0,403 \mathrm{~g} \mathrm{~cm}^{-3}$ de densidade básica média para indivíduos de quatro anos de idade no município de Colombo, PR.

A densidade energética relaciona-se diretamente com a energia contida em um determinado volume de madeira. O valor observado neste trabalho foi de 2.206,9 Mcal m (Tabela 1), semelhante aos valores relatados por Lima et al. (2011), para árvores de seis anos de idade (2.222,0 $\left.\mathrm{Mcal} \mathrm{m}^{-3}\right)$.

$O$ PCI médio da madeira de $E$. benthamii determinado foi de 4.379, $1 \mathrm{kcal} \mathrm{kg}^{-1}$ e o PCS foi de 4.679,7 $\mathrm{kcal} \mathrm{kg}^{-1}$ (Tabela 1). Lima et al. (2011) observaram um valor desta variável de 4.681,0 $\mathrm{kcal} \mathrm{kg}^{-1}$ na região de Guarapuava, PR. Na análise de correlação de Pearson (Tabela 3) observou-se que o PCS se correlacionou negativamente com o teor de materiais voláteis $(-0,3857)$, ou seja, quanto maior a quantidade de materiais voláteis na madeira, menor será o poder calorífico. O mesmo resultado foi observado por Reis et al. (2012) que relataram uma correlação negativa entre estas duas variáveis no carvão de E. urophylla S.T. Blake.

A composição química imediata refere-se ao teor de carbono fixo, materiais voláteis e cinzas presentes na massa da madeira. Segundo Brand (2010) os teores de materiais voláteis e carbono fixo são inversamente proporcionais. Isto pode ser observado na análise de correlação entre o teor de materiais voláteis e o teor de carbono fixo, que apresentou valor negativo $(-0,9930)$ (Tabela 3). Pelo conhecimento do índice percentual de materiais voláteis, pode-se estimar o grau de combustão de um combustível, ou seja, combustíveis com altos índices de carbono fixo (baixo índice de voláteis) queimarão mais lentamente.

A biomassa com maior teor de materiais voláteis e menor teor de cinzas apresenta, normalmente, menor poder calorífico superior. Os valores médios obtidos na madeira de carbono fixo, materiais voláteis e teores de cinzas foram, respectivamente, $15,78 \%, 84,18 \%$ e $0,24 \%$ (Tabela 1). Brito \& Barrichelo (1978) realizaram a análise química imediata de diferentes espécies eucalipto, sendo que as espécies que mais se aproximaram dos valores encontrados para $E$. benthamii foram $E$. saligna, que apresentou $16,7 \%$ de carbono fixo, E. tereticornis e $E$. resinifera, com $84,2 \%$ de materiais voláteis, $E$. resinifera e E. camaldulensis, com $0,30 \%$ de teor de cinzas.

A análise química da madeira de E. benthamii apresentou valor médio de carbono (46,68\%) (Tabela 3 ) semelhante ao valor médio de E. urophylla (46,76\%) encontrado por Reis et al. (2012).

O mesmo padrão foi observado para o teor de hidrogênio, que para $E$. benthamii foi de 5,94\% (Tabela 3 ) e para E. urophylla foi de $6,14 \%$, em estudo de Reis et al. (2012). Reis et al. (2012) relataram que o nitrogênio não apresenta relação positiva com o poder calorífico superior. Comparando-se as substâncias que constituem a parede celular das plantas, é importante ressaltar que a lignina é rica em carbono e hidrogênio, que são os elementos que produzem calor e, portanto, apresentam poder calorífico maior que os carboidratos (Brand, 2010). Barcellos et al. (2005) encontraram 0,30\% de nitrogênio para o gênero Eucalyptus, valor menor comparado ao encontrado neste trabalho para $E$. benthamii de $1,63 \%$.

A relação $\mathrm{C} / \mathrm{H}$ observada $(7,87)$ foi semelhante ao encontrado por Reis et al. (2012) em E. urophylla $(7,62)$. Por outro lado, a relação $\mathrm{C} / \mathrm{N}$ encontrada $(29,23)$ foi inferior à relatada pelos mesmos autores para $E$. urophylla $(442,07)$, onde mencionam que elevada razão $\mathrm{C} / \mathrm{N}$ implica em menor quantidade de nitrogênio que será liberada para o ambiente após a combustão da biomassa.

\section{Conclusões}

O potencial energético da madeira de $E$. benthamii não é modificado ao longo das diferentes alturas do fuste, não havendo diferença nos valores de densidade básica e energética, composição química imediata e poder calorífico superior e inferior.

O poder calorífico superior é positivamente correlacionado com o poder calorífico inferior e com a densidade básica. É também correlacionado com o teor de materiais voláteis, carbono fixo e cinzas, porém, negativamente. 
As características energéticas da madeira de $E$. benthamii indicam potencial para uso como matériaprima na geração de energia.

\section{Referências}

ASSOCIAÇÃO BRASILEIRA DE NORMAS TÉCNICAS. NBR 8112: análise imediata: material volátil, cinzas, carbono fixo. Rio de Janeiro, 1983. 6 p.

ASSOCIAÇÃO BRASILEIRA DE NORMAS TÉCNICAS. NBR 8633: carvão vegetal: determinação do poder calorífico. Rio de Janeiro, 1984. 13 p.

ASSOCIAÇÃO BRASILEIRA DE NORMAS TÉCNICAS. NBR 11941: madeira: determinação da densidade básica. Rio de Janeiro: 2003. $6 \mathrm{p}$.

BARCELlOS, D. C.; COUTO, L. C.; MÜLlER, M. D.; COUTO, L. O estado-da-arte da qualidade da madeira de eucalipto para a produção de energia: um enfoque nos tratamentos silviculturais.

Biomassa \& Energia, v. 2, n. 2, p. 141-158, 2005.

BRAND, M. A. Energia de biomassa florestal. Rio de Janeiro: Interciência, 2010. $131 \mathrm{p}$.

BRITO, J. O.; BARRICHELO, L. E. G. Características do eucalipto como combustível: análise química imediata da madeira e da casca.

IPEF, Piracicaba, n. 16, p. 63-70, 1978.
CERRO NEGRO (Prefeitura municipal). Informações gerais de Cerro Negro. 2015. Disponível em: $<$ http://www.cerronegro.sc.gov. br/cms/pagina/ver/codMapaItem/67406>. Acesso em: 29 out. 2015.

DOAT, J. Le pouvoir calorifique des bois. Revue Bois et Forêsts des Tropiques, n. 172, p. 33-55, 1977.

HIGA, R. C. V.; PEREIRA, J. C. D. Usos potenciais do Eucalyptus benthamii Maiden et Cambage. Colombo: Embrapa Florestas, 2003. 4 p. (Embrapa Florestas. Comunicado técnico, 100).

LIMA, E. A.; SILVA, H. D.; LAVORANTI, O. J. Caracterização dendroenergética de árvores de Eucalyptus benthamii. Pesquisa Florestal Brasileira, Colombo, v. 31, n. 65, p. 09-17, jan./mar. 2011. DOI: $10.4336 / 2010 . p f b .31 .65 .09$.

NISGOSKI, S.; MUNIZ, G. I. B.; KLOCK, U. Caracterização anatômica da madeira de Eucalyptus benthamii Mainden et Cambage. Ciência Florestal, Santa Maria, RS, v. 8, n. 1, p. 67-76, 1998.

PEREIRA, J. C. D.; STURION, J. A.; HIGA, A. R.; HIGA, R. C. V.; SHIMIZU, J. Y. Características da madeira de algumas espécies de eucalipto plantadas no Brasil. Colombo: Embrapa Florestas, 2000. 113 p. (Embrapa Florestas. Documentos, 38).

QUIRINO, W. F.; VALE, A. T.; ANDRADE, A. P. A.; ABREU, V. L. S.; AZEVEDO, A. C. S. Poder calorífico da madeira e de resíduos ligno-celulósicos. Biomassa \& Energia, v. 1, n. 2, p. 173-182, 2004.

REIS, A. A.; PROTÁSIO, T. P.; MELO, I. C. N. A.; TRUGILHO, P. F.; CARNEIRO, A. C. O. Composição da madeira e do carvão vegetal de Eucalyptus urophylla em diferentes locais de plantio. Pesquisa Florestal Brasileira, Colombo, v. 32, n. 71, p. 277-290, 2012. DOI: 10.4336/2012.pfb.32.71.277. 
